\title{
A Propeller Flap for Single-Stage Nose Reconstruction in Selected Patients: Supratrochlear Artery Axial Propeller Flap
}

\author{
Adriana Cordova, MD ${ }^{1}$ Salvatore D’Arpa, MD, $\mathrm{PhD}^{1}$ \\ Francesca Toia, MD $^{1}$ Francesco Moschella, MD ${ }^{1}$ \\ ${ }^{1}$ Chirurgia Plastica e Ricostruttiva, Dipartimento di Discipline \\ Chirurgiche, Oncologiche e Stomatologiche, Università degli Studi di \\ Palermo, Palermo, Italy
}

Tripoli Massimiliano, MD, PhD ${ }^{1}$

\begin{abstract}
Address for correspondence Adriana Cordova, MD, U.O. di Chirurgia Plastica e Ricostruttiva, A.O.U.P. Paolo Giaccone, Via del Vespro 129, 90127 Palermo, Italy (e-mail: adriana.cordova.unipa@gmail.com).
\end{abstract}

\begin{abstract}
Keywords

- nose reconstruction

- propeller flap

- forehead flap

- supratrochlear artery

The paramedian forehead flap is the gold standard technique for nose reconstruction. It requires two different surgical operations which prolonged the postoperative dressing and care. We present our 5-year experience with a propeller flap based on the supratrochlear artery, which allows one-stage transfer of the forehead skin to the nose without the need for pedicle division. This technique is indicated in a selected group of patients who are not suitable for multiple-stage reconstructions because they have concurrent medical conditions, reduced mobility, or live far away from specialized medical centers. We have renamed this procedure as supratrochlear artery axial propeller flap, from the acronym STAAP flap, to stress the axial, well known and constant, vascularization of the flap. In the past 5 years, we have been performing 25 STAAP flaps; full-thickness nasal reconstruction was performed in 11 cases. The patients were 16 males and 9 females, with a mean age of 79.5 years. All patients had multiple comorbidities. Complete flap survival was observed in 23 cases and healing was complete in 7 days. In two cases, there was a partial distal necrosis of the flap treated conservatively. Cosmetic results were good and the patient's satisfaction was significant. These results indicate that the STAAP flap is a reliable and useful technique in selected cases, as old or noncompliant patients who benefit from a one-stage technique of nose reconstruction.
\end{abstract}

The paramedian forehead flap for nose reconstruction gives excellent cosmetic results, but it needs multiple surgical procedures and prolonged postoperative dressing and care. ${ }^{1,2}$ In selected cases, such as elderly, noncompliant patients and patients living far away from specialized medical centers, a single-stage procedure for nose reconstruction can be desirable. In this article, we present our experience using a forehead propeller flap to perform a one-stage nose reconstruction.

Today, we indicate this innovation of the forehead flap as "supratrochlear artery axial propeller flap," from the acronym STAAP flap. ${ }^{3}$
The STAAP flap is pedicled on the supratrochlear artery, a terminal branch of the ophthalmic artery, 1 of the 10 main source arteries in the head and neck. The ophthalmic artery is a terminal branch of the internal carotid artery that exits the cranial vault via the optic canal. The territory of the ophthalmic artery includes the skin of the forehead and dorsum of the nose. Its terminal branches are the medial palpebral arteries (superior and inferior arches), the lacrimal artery (that has no cutaneous perforators), the inferior palpebral arch, and the supraorbital and supratrochlear arteries; the supraorbital and
Issue Theme Nasal Reconstruction; Guest Editor, Helmut Fischer, MD
Copyright @ 2014 by Thieme Medical Publishers, Inc., 333 Seventh Avenue, New York, NY 10001, USA. Tel: +1(212) 584-4662.
DOI http://dx.doi.org/ 10.1055/s-0034-1376877. ISSN 0736-6825. 
supratrochlear arteries have been used as the vascular axis for many pedicled forehead flaps for nasal reconstruction.

The supratrochlear artery, together with its accompanying veins and the supratrochlear nerve, emerges from the concavity of the inner canthus, perforates the procerus and corrugator supercilii muscles, and runs within the frontalis muscle to nourish the paramedian forehead skin. ${ }^{4}$

The STAAP has the same pedicle of a classical forehead flap but, thanks to "perforator like" dissection of its pedicle, can be mobilized like a propeller flap getting the possibility to rotate 180 degrees. ${ }^{5,6}$

The STAAP must not be considered an alternative to the conventional multistage forehead flap, but rather an alternative flap indicated when a single-stage procedure and a fast recovery have priority over a perfect cosmetic result with subunit reconstruction.

\section{Surgical Technique}

Once the defect to be repaired has been outlined, a hand held Doppler probe is used to identify the exact point where the supratrochlear artery emerges. This point is marked on the skin (-Figs. 1 and $\mathbf{2}$ ).

The flap is planned as a skin island, centered on this point, composed by two blades, like a propeller: a superior or frontal blade and an inferior or nasal blade. The frontal blade is planned as is usually done for a conventional forehead flap, with the aid of a template of the recipient site defect (-Fig. 2).

The nasal blade includes the skin between the supratrochlear artery and the upper margin of the defect. Precise planning will allow to plan a flap as narrow as possible at the pivot point, to avoid undesirable folds. Dissection is performed in the supraperiosteal plane, besides in the distal part of the frontal blade where it is done supramuscular (-Fig. 3), like in a conventional forehead flap. When the emergence of the pedicle is approximated, dissection is

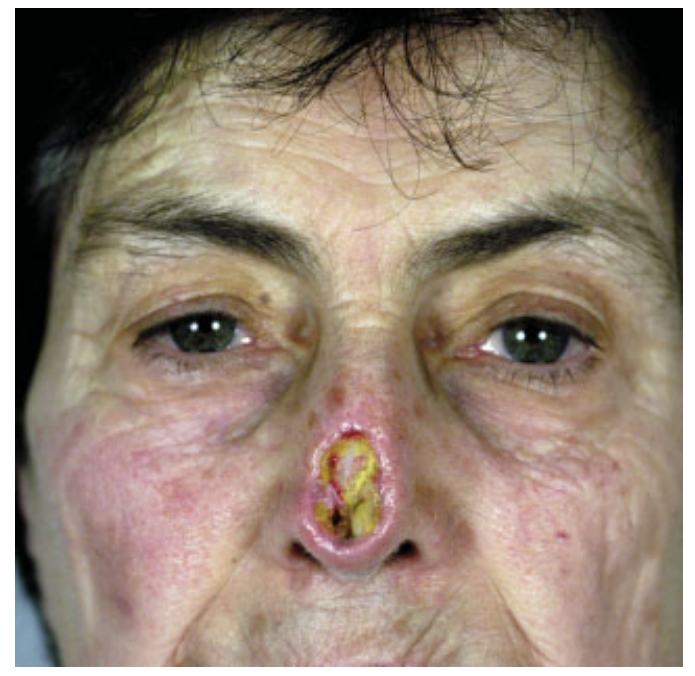

Fig. 1 One week after resection of an ulcerated basal cell carcinoma of the tip in a 69-year-old female. Reconstruction was delayed until final pathology was available.

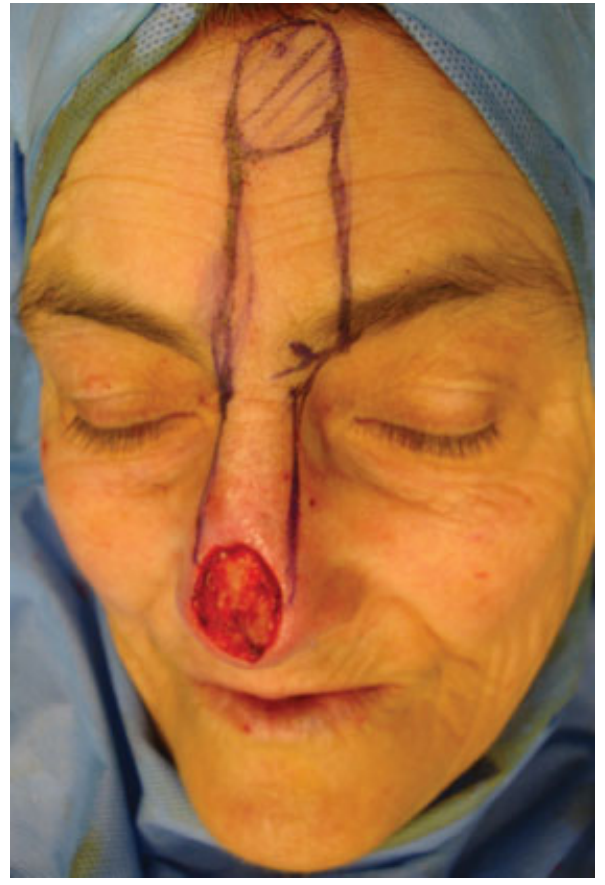

Fig. 2 A long flap is planned. The striped area corresponds to the defect. All the skin between this area and the defect must be harvested. The dorsal nasal skin does not necessarily have to be preserved with the flap.

performed with caution using loupe magnification. The vessels have to be identified and freed from any attachment to the surrounding tissue that might impair pedicle rotation. As the pedicle is approached, muscular attachments of the corrugator supercilii ( - Fig. 4) will have to be divided and the vessels will come into view. Meticulous hemostasis using bipolar forceps is mandatory to maintain a bloodless field.

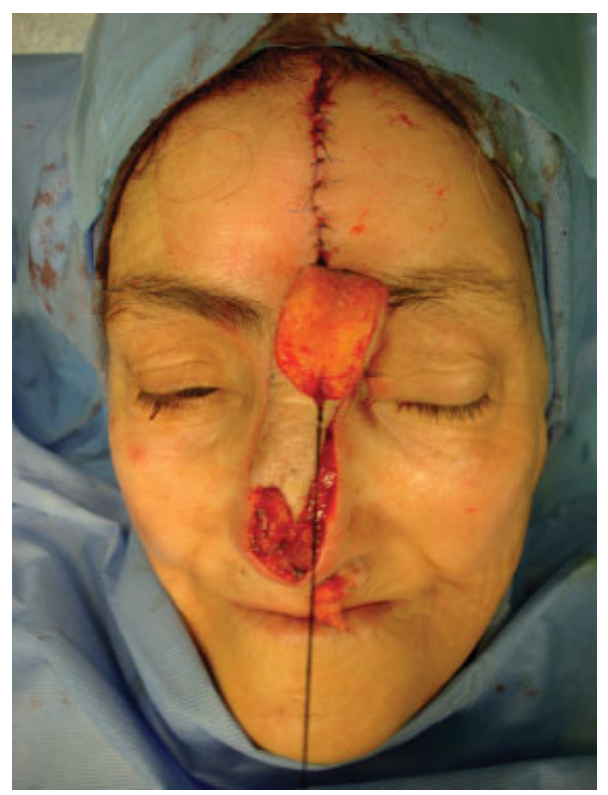

Fig. 3 Like in a conventional forehead flap, the distal $1.5 \mathrm{~cm}$ is harvested without muscle to obtain a thinner flap. 


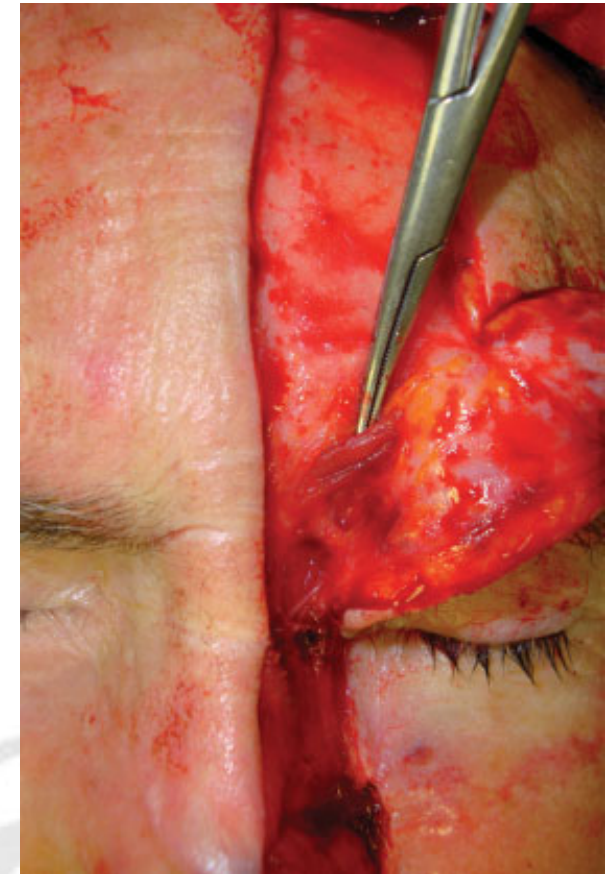

Fig. 4 During supraperiosteal craniocaudal dissection, when the pedicle is approached the fibers of the corrugator supercilii muscle are encountered. After these fibers are divided the pedicle is identified.

Once the pedicle is identified and marked with a vessel loop, the flap can be cut circumferentially and dissection will be performed for the nasal blade to obtain exposure of the nasal side of the pedicle. The pedicle, if necessary, can be isolated until its emergence from the frontal bone. In this case it must be then freed from its periosteal adhesion and its side

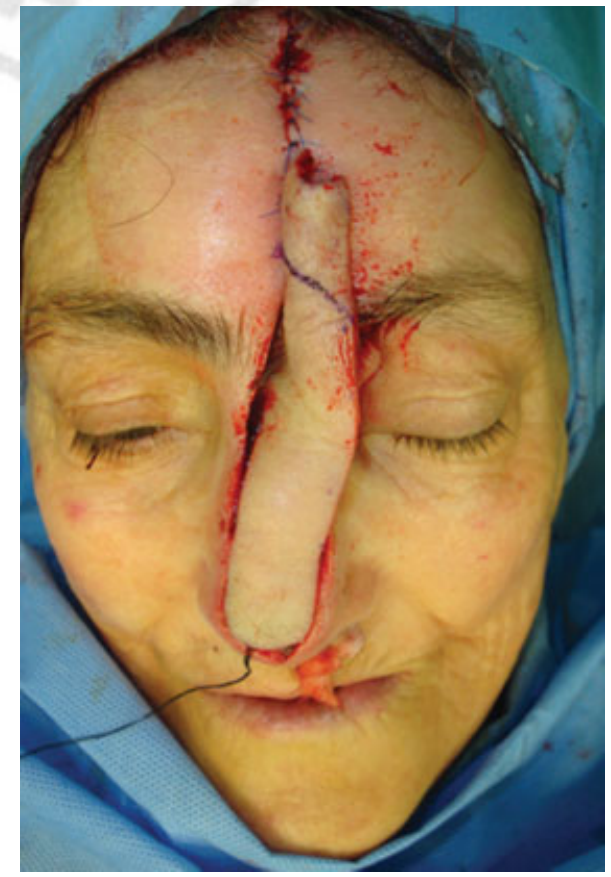

Fig. 5 After rotation, the nasal blade is too long and is not needed in the forehead. The portion to be discarded is marked.

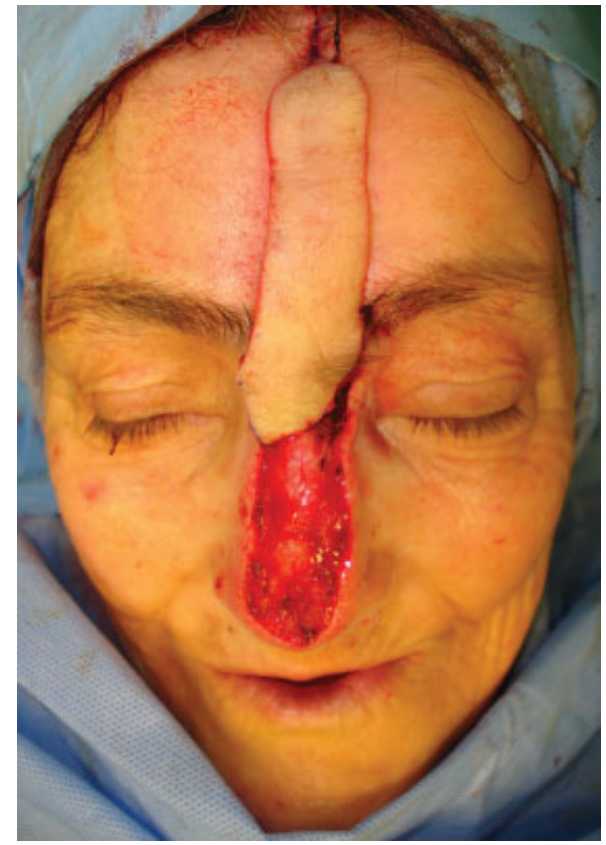

Fig. 6 The dorsal nasal skin has been discarded except for a small part that will be positioned between the eyebrows to protect the pedicle and maintain interbrow distance.

branches, one going toward the lateral brow and one laterally to the upper eyelid, cauterized or better ligated with a 5 or $6 / 0$ thread and cut to avoid twisting or kinking of the vascular pedicle during flap transfer. Some nervous branches are identified at this point too. They must be cauterized and cut to allow pedicle rotation. At this point, the flap is only attached by its pedicle and the pedicle is free from any attachment to the surrounding tissues. It is not uncommon that a flap has a purplish hue or is paler than the surrounding skin. These temporary vascular disturbances are due to spasm caused by pedicle dissection. Xylocaine irrigation and letting the flap rest for a few minutes before rotation will let them disappear. Meanwhile, the donor site can be approximated (-Figs. 3, 5, and 6) and cartilage grafts, flaps for the inner lining or other procedures performed. Rotation is simulated both clockwise and counterclockwise and perfusion of the skin and pedicle kinking are checked to decide which rotational direction is better. Rotation is 180 degrees in both senses anyway. However, inward rotation (counterclockwise for left-sided flaps and clockwise for right-sided flaps) is commonly the best sense of rotation. Once the flap is rotated, the frontal blade covers the loss of substance of the nose like a conventional forehead flap, while the nasal blade is used to partially cover the donor site defect. Alternatively, when using the nasal blade for donor site closure is not advantageous, it can be discarded (-Figs. 4-8) or used for the lining if a full-thickness reconstruction has to be performed (-Figs. 9-16). Full-thickness defects of the nasal ala can also be repaired by planning a longer frontal blade and folding it double. Otherwise, septal flaps or composite grafts, to repair inner layer, can be successfully used in the same operation. 


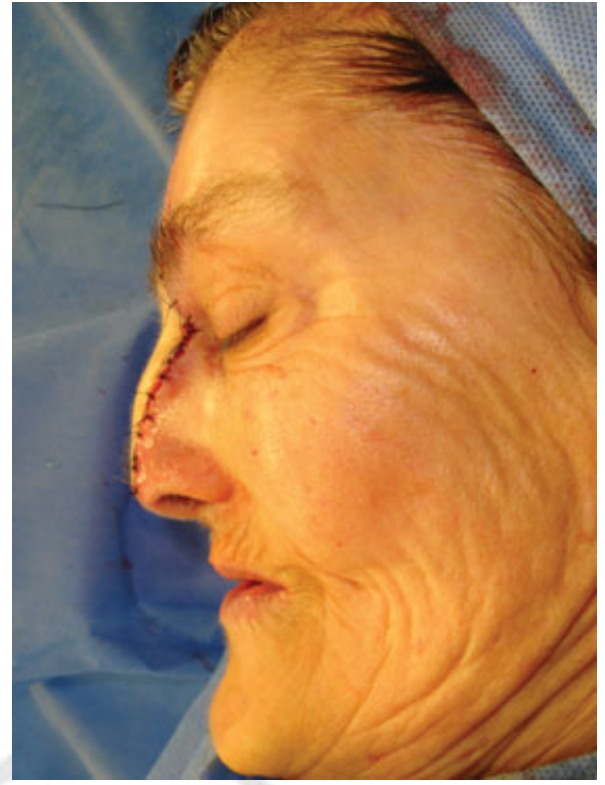

Fig. 7 Immediate postoperative lateral view. Note the preservation of nasal contour.

\section{Patients and Results}

Nose reconstruction by means of the STAAP flap was performed on 25 patients after excision of cutaneous malignancies, 14 basal cell carcinomas (BCCs), 10 squamous cell carcinomas (SCCs), and 1 melanoma (M). The patients were 16 males and 9 females. Ages ranged between 67 and 90 (mean 79.5). All patients had some kind of comorbidity: hypertension $100 \%$, type II diabetes $60 \%$, liver cirrhosis $2 \%$, and smoking history $90 \%$. Full-thickness reconstruction was performed on 11 patients ( $1 \mathrm{BCC}, 1 \mathrm{M}$, and 9 SCCs). Defects of the nose skin involved: 2 cases the entire surface of the nose enclosed nostrils, 8 cases the skin of the dorsum without the tip, 5 cases the distal subunits (tip and alae), and 10 cases of full-thickness defects of the ala and lateral nasal wall (-Fig. 17). Different options were used to reconstruct the

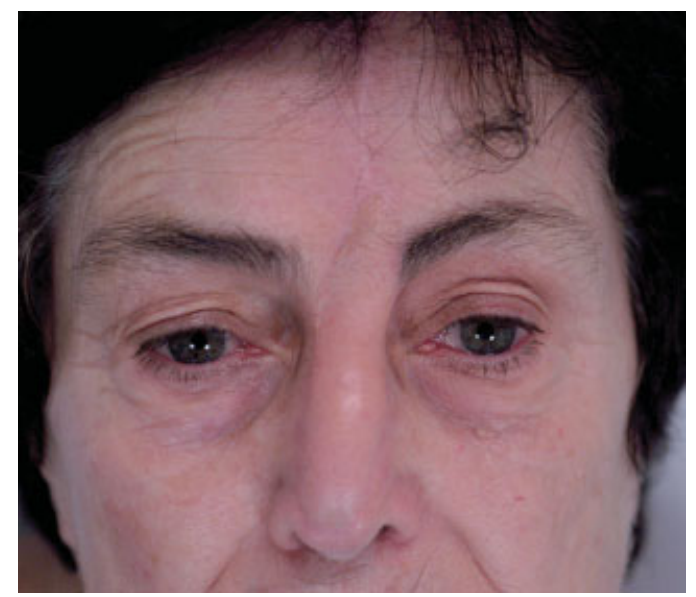

Fig. 8 One year postoperative result of a one-stage operation.

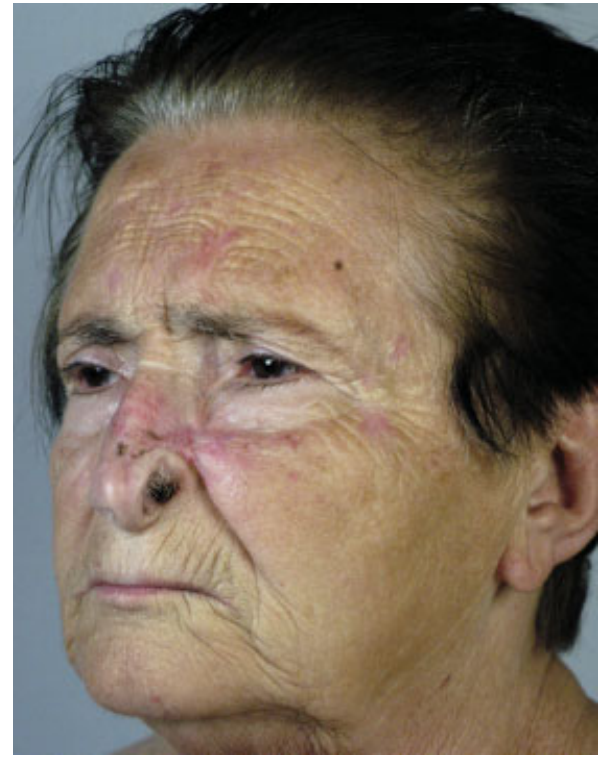

Fig. 9 Recurrent squamous cell carcinoma of the dorsum and left ala in a 77-year-old patient. The cancer and the previous operations have caused retraction of the nostril margin.

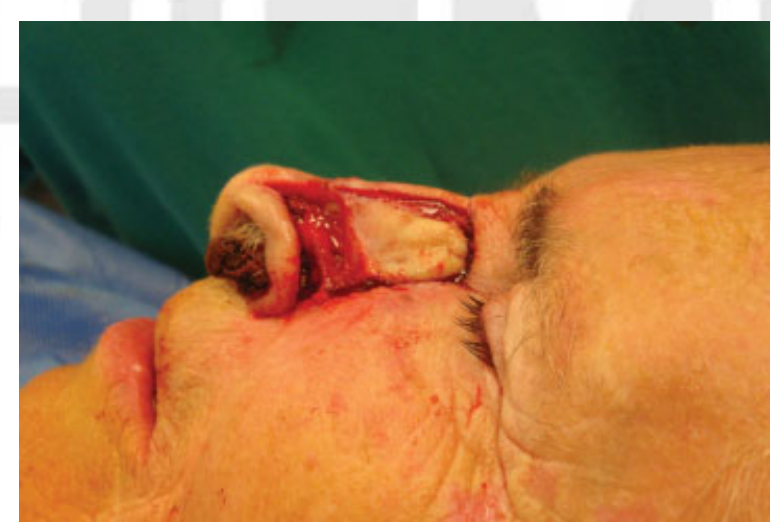

Fig. 10 After full-thickness tumor resection, the nostril margin is released and put back in place. A trapdoor flap is harvested from the healthy dorsum based on the margin of the defect.

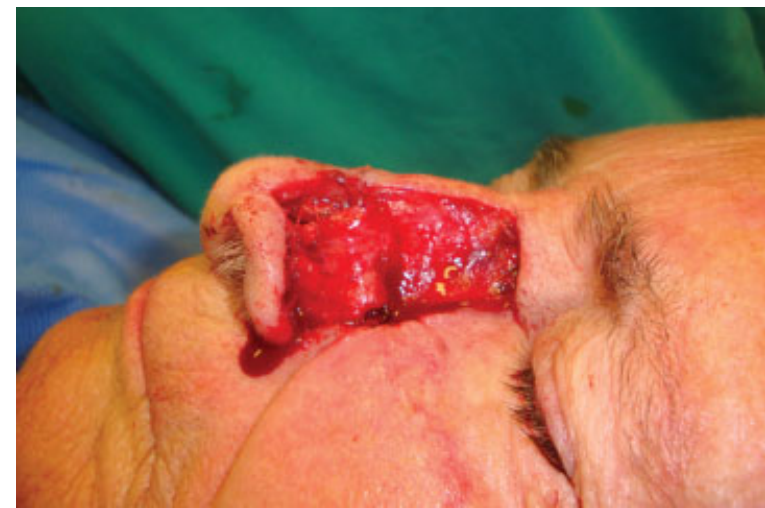

Fig. 11 The flap is revolved to replace the inner lining. 


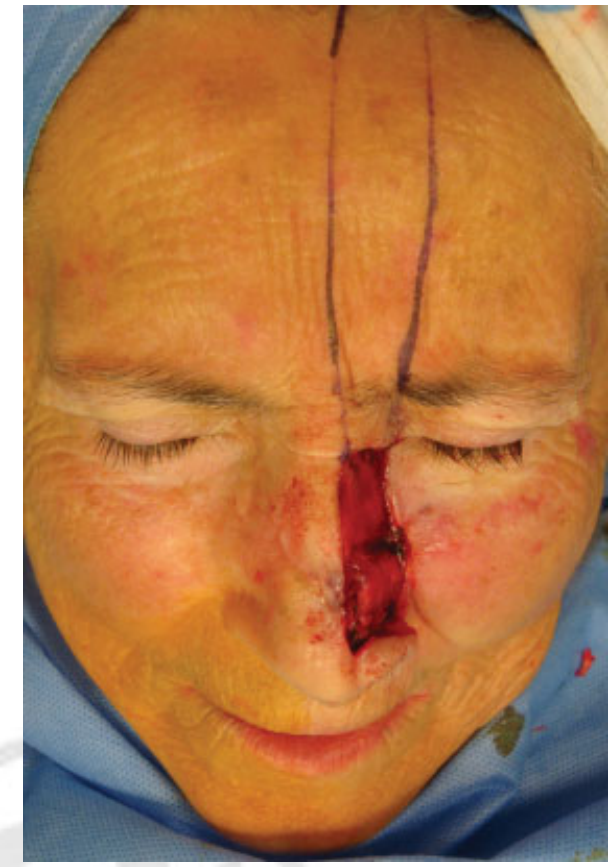

Fig. 12 A long and narrow STAAP flap is drawn on the skin. Especially, the base should be as narrow as possible to preserve profile of the glabellar area. STAAP, supratrochlear artery axial propeller.

internal lining. We used a composite chondrocutaneous graft $^{7}$ for full-thickness defects of the nasal ala, in four cases, and for major defects of the dorsum and lateral cartilage, in one case. In three cases to repair losses of substances of the dorsum, without loss of skeletal support, the residual dorsal

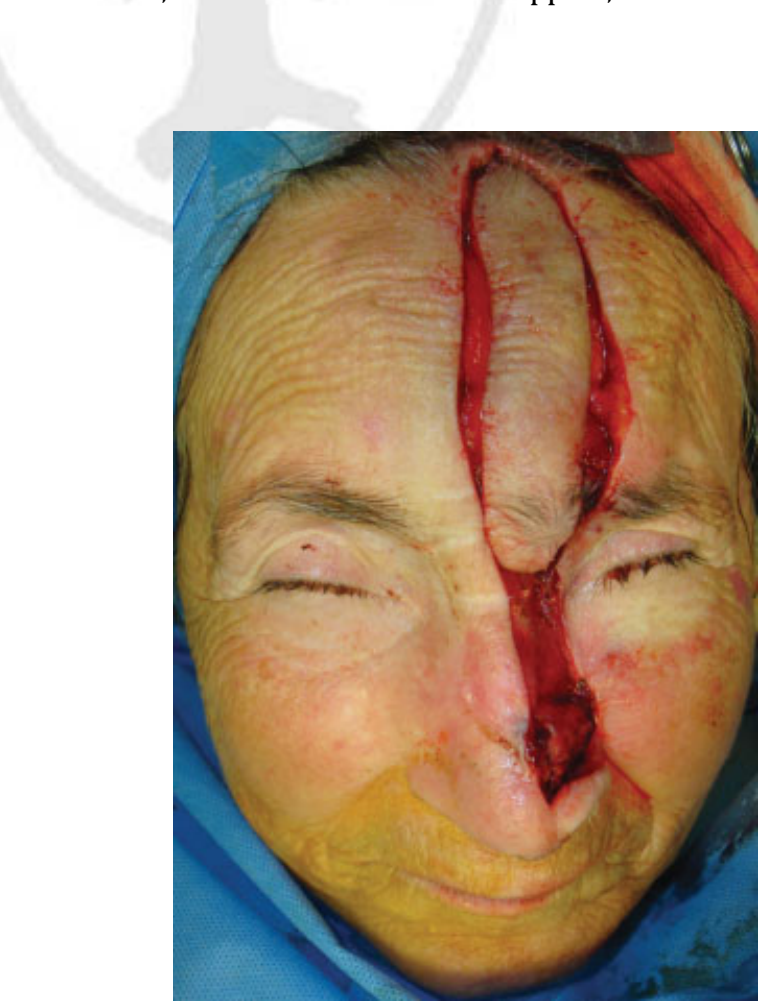

Fig. 13 The flap after harvest has good color. To avoid dog ears, the incision is extended into the hair bearing skin, but the hairy portion of the flap will be discarded.

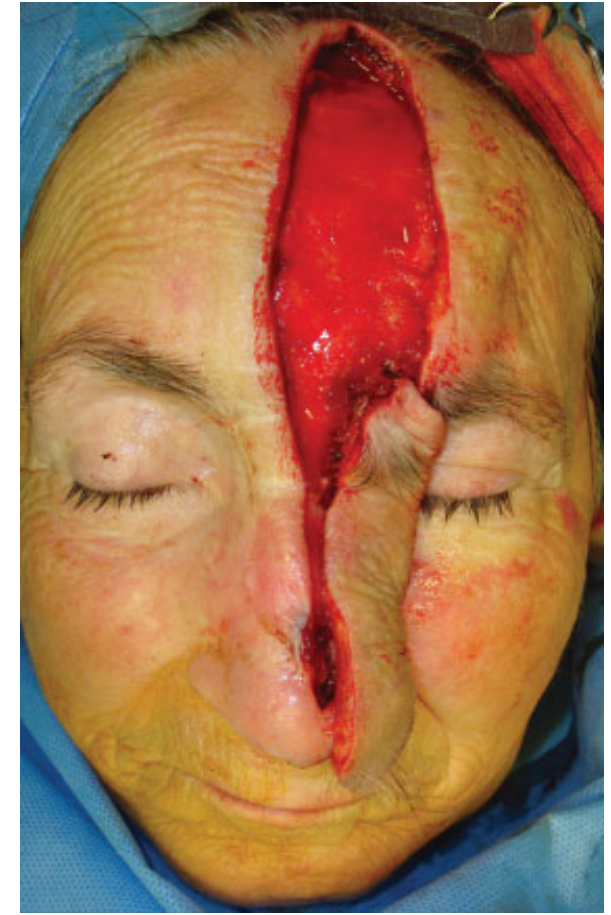

Fig. 14 The flap is longer than the defect. Shortening should leave a little longer to avoid traction on the pedicle or on the nostril.

nasal skin was revolved and lower pedicled. In three cases of full-thickness defects of the ala, with good skeletal support, the distal portion of the frontal blade was thinned and folded double to repair the mucosal defect. Patients' data are

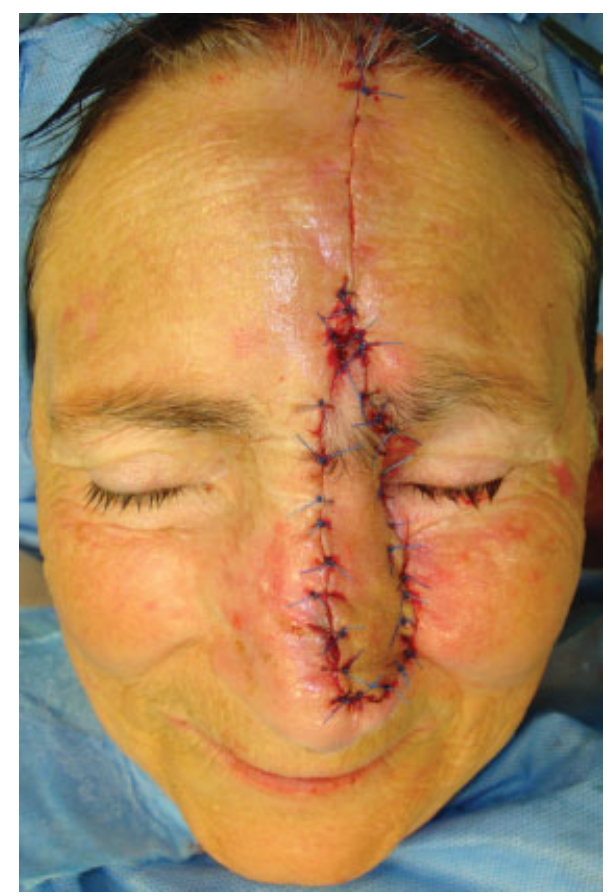

Fig. 15 At the end of the operation, continuity of the brow is restored: the growth direction of the hair is inverted and now points downward. 


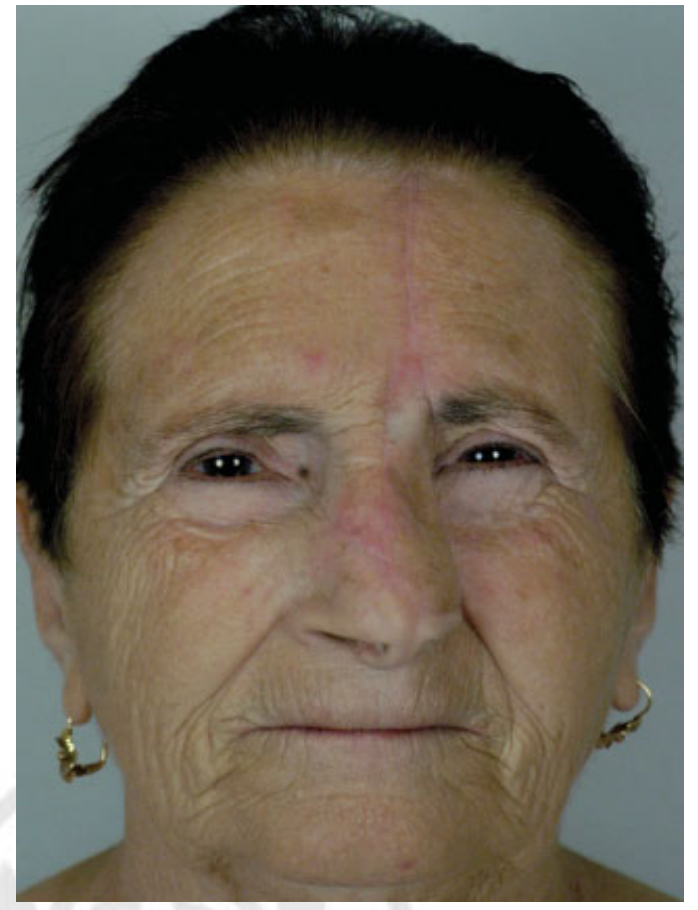

Fig. 16 One year postoperative frontal view. The preoperative tip deviation to the left was not corrected by the operation. The patient had shaved the medial eyebrow to achieve symmetry.

resumed in - Table 1 . All flaps were rotated 180 degrees and 23 flaps (92\%) healed uneventfully (-Figs. 18-21), despite a temporary postoperative venous congestion was sometimes observed (-Figs. 22-27). One flap had partial necrosis (4\%). One patient underwent a second operation for thinning the flap at the glabella to optimize the cosmetic outcome. All the operations were performed under local anesthesia.

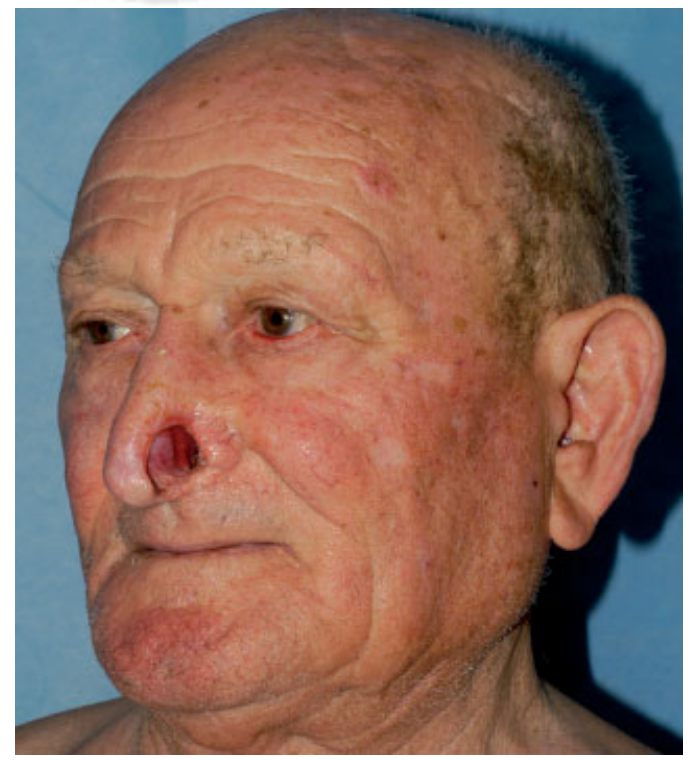

Fig. 17 Alar and lateral wall defect after full-thickness resection of SCC in a 79-year-old male, without reconstruction. SCC, squamous cell carcinoma.

\section{Discussion}

A single-stage operation for nose reconstruction with the forehead skin is a goal that plastic surgeons have always tried to pursue over the years. The STAAP combine the design of a conventional forehead flap with the "perforator like" dissection of the pedicle and the "propeller rotation" of the skin.

This technical innovation allows us to obtain a one-stage reconstruction of the nose with all advantages of an axial flap and the mobility of a propeller. Perforator flaps have already allowed us to achieve good results in one-stage nose reconstructions. ${ }^{8,9}$ In the light of that experience, we have started using the STAAP flap if a speedy recovery was required.

Converse and Wood-Smith ${ }^{10}$ described in 1963 a singlestage island flap from the forehead. This flap had several problems such as flap congestion, inadequate reach, and marked fullness along the glabella. The authors abandoned this design early. Recently, Park in $2002^{11}$ recovered the project of a single-stage nasal reconstruction proposing a midline forehead island flap with a unilateral subcutaneous pedicle. Mombaerts and Gillies proposed a similar technique for reconstruction of the medial canthus and lower eyelids. ${ }^{12}$ We have used Park's technique in four cases, in two cases we had an acceptable result, but in two cases (50\%) flap congestion and partial necrosis was observed. In Park's technique, the flap is tunneled under the glabellar skin and this can create congestion of the flap and fullness in the glabellar area. Moreover, the author recommends this technique only for defects limited to the upper two-thirds of the nose and without risk factors such as peripheral vascular disease, coronary disease, hypertension, diabetes, and previous radiotherapy. Elderly patients, however, the subset of patients most commonly affected by cutaneous malignancies, often have various such comorbidities and, according to Park himself, cannot be candidates to the technique. Techniques of one-stage nose reconstruction have failed to achieve popularity because of the above-mentioned drawbacks. Menick, after having gained remarkable experience, has described a three-stage forehead flap for nose reconstruction, adding an additional stage for flap remodeling. The threestage technique allows to achieve the best cosmetic result so far, but it necessitates prolonged wound care (the pedicle of the flap can bleed during flap delay), the inability to wear eyeglasses (often a necessity for the elderly) and the disturbing dressing on the nose that they have to wear for 6 weeks at best. All this drawbacks of the conventional flap are, however, very well tolerated by many patients who have high motivations and cosmetic demands. Conversely, patients who are in advanced age are less prone to withstand multiple surgical stages and, at least in our experience, often refuse complex reconstructive methods even at the expense of cosmetic result. Also, in major hospitals like our university hospital, patients living far away are often treated. For these patients, a technique that gives a good result with a fast recovery might be preferable over one that gives an excellent result but with multiple operations and prolonged wound care. STAAP flap is a simple and safe technique in the hands of surgeons which 
Table 1 Data of the patients who underwent STAAP reconstruction

\begin{tabular}{|c|c|c|c|c|c|c|c|}
\hline Case & Sex & Age & Pathology & $\begin{array}{l}\text { Nasal } \\
\text { subunit }\end{array}$ & Excision & $\begin{array}{l}\text { Inner layer } \\
\text { reconstruction }\end{array}$ & Complications \\
\hline 1 & $\mathrm{M}$ & 67 & BCC & Dorsum & Partial-thickness reconstruction & & \\
\hline 2 & M & 72 & SCC & Ala + lateral wall & Full-thickness reconstruction & & \\
\hline 3 & $\mathrm{~F}$ & 69 & $\mathrm{BCC}$ & Tip and ala & Partial-thickness reconstruction & & \\
\hline 4 & $\mathrm{~F}$ & 71 & SCC & Ala + lateral wall & Full-thickness reconstruction & $\begin{array}{l}\text { Composite } \\
\text { chondrocutaneous } \\
\text { graft }\end{array}$ & \\
\hline 5 & $\mathrm{M}$ & 84 & SCC & Tip and ala & Partial-thickness reconstruction & Revolved dorsal skin & \\
\hline 6 & M & 72 & BCC & Ala + lateral wall & Full-thickness reconstruction & Distal folded flap & \\
\hline 7 & $\mathrm{M}$ & 81 & $\mathrm{BCC}$ & Dorsum & Partial-thickness reconstruction & & \\
\hline 8 & $\mathrm{M}$ & 79 & $\mathrm{BCC}$ & Dorsum & Partial-thickness reconstruction & & \\
\hline 9 & $\mathrm{~F}$ & 74 & SCC & Ala + lateral wall & Full-thickness reconstruction & Distal folded flap & \\
\hline 10 & M & 89 & $\mathrm{BCC}$ & Dorsum & Partial-thickness reconstruction & & \\
\hline 11 & $\mathrm{M}$ & 83 & SCC & Ala + lateral wall & Full-thickness reconstruction & Revolved dorsal skin & \\
\hline 12 & $\mathrm{~F}$ & 76 & SCC & Ala + lateral wall & Full-thickness reconstruction & $\begin{array}{l}\text { Composite } \\
\text { chondrocutaneous } \\
\text { graft }\end{array}$ & \\
\hline 13 & M & 82 & $\mathrm{BCC}$ & Dorsum & Partial-thickness reconstruction & & \\
\hline 14 & $\mathrm{~F}$ & 77 & SCC & Ala + lateral wall & Full-thickness reconstruction & $\begin{array}{l}\text { Composite } \\
\text { chondrocutaneous } \\
\text { graft }\end{array}$ & \\
\hline 15 & $\mathrm{~F}$ & 75 & SCC & Dorsum & Full-thickness reconstruction & $\begin{array}{l}\text { Composite } \\
\text { chondrocutaneous } \\
\text { graft }\end{array}$ & \\
\hline 16 & $\mathrm{M}$ & 79 & SCC & Ala + lateral wall & Full-thickness reconstruction & $\begin{array}{l}\text { Composite } \\
\text { chondrocutaneous } \\
\text { graft }\end{array}$ & Partial necrosis \\
\hline 17 & $\mathrm{~F}$ & 72 & $\mathrm{BCC}$ & Tip and ala & Partial-thickness reconstruction & & \\
\hline 18 & $\mathrm{M}$ & 74 & SCC & Ala + lateral wall & Full-thickness reconstruction & Revolved dorsal skin & \\
\hline 19 & M & 85 & $\mathrm{BCC}$ & Dorsum & Partial-thickness reconstruction & & \\
\hline 20 & M & 79 & $\mathrm{BCC}$ & Tip and ala & Partial-thickness reconstruction & & \\
\hline 21 & $\mathrm{~F}$ & 90 & $\mathrm{BCC}$ & Entire surface & Partial-thickness reconstruction & & \\
\hline 22 & $\mathrm{~F}$ & 80 & M & Ala + lateral wall & Full-thickness reconstruction & Distal folded flap & \\
\hline 23 & $\mathrm{M}$ & 74 & $\mathrm{BCC}$ & dorsum & Partial-thickness reconstruction & & \\
\hline 24 & M & 86 & $\mathrm{BCC}$ & Tip and ala & Partial-thickness reconstruction & & \\
\hline 25 & $\mathrm{M}$ & 73 & BCC & Entire surface & Partial-thickness reconstruction & & \\
\hline
\end{tabular}

Abbreviations: BCC, basal cell carcinoma; M, melanoma; SCC, squamous cell carcinoma; STAAP, supratrochlear artery axial propeller.

are used to microsurgical techniques and to raising perforator propeller flaps.

The dissection of the supratrochlear pedicle, at its emergence, requires intramuscular dissection and loupe magnification; for this reason, in our first publication, ${ }^{13}$ we named this flap STAPP to underline the "perforator like" dissection of the pedicle. Today, we prefer the acronym STAAP to point out the type of vascularization of this flap. The nomenclature of the flap was chosen looking to the nomenclature proposed by Geddes et al. First, the name of the artery enclosed in the pedicle (supratrochlear artery). The second A mean "Axial" and it indicates the axial vascular pattern of this flap. The $P$ indicates that this is definitely a propeller flap because of the shape of the flap: an island flap, raised and rotated like a "propeller" with two blades. ${ }^{14}$ Supratrochlear artery is a vessel constant for caliber and site, and it is one of the terminal branches of the ophthalmic artery, it passes through the muscles procerus and corrugator of eyebrows but is not a perforator. The STAAP flap can reach any kind of nasal defects with good results. It can repair losses of substance of the 


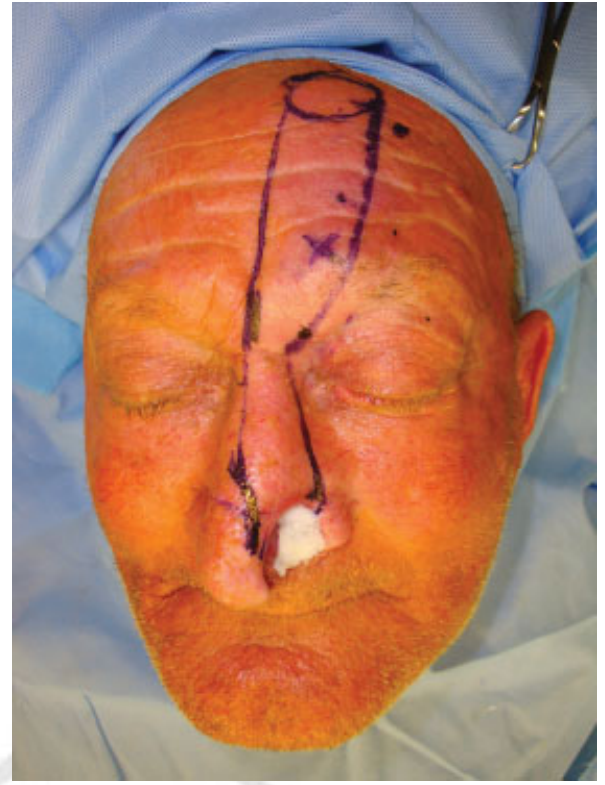

Fig. 18 Preoperative marking. A very long flap is needed to fold it around the nostril margin. In a bald patient, this will not bring any unnecessary hair into the nose. The " $X$ " marks a Doppler signal that does not correspond to the exit foramen of the pedicle. The sound was heard too high. Note that the narrowest portion of the flap is around the perforator.

upper third of the nose and it can be long enough to repair distal defects, folded to reconstruct the nasal margin. The nasal blade of the propeller repairs the glabellar area and preserves the normal distance between the brows, with scars overlapping with the glabellar groves. No harm is done to the patient by elevating this area even if it is discarded, similarly

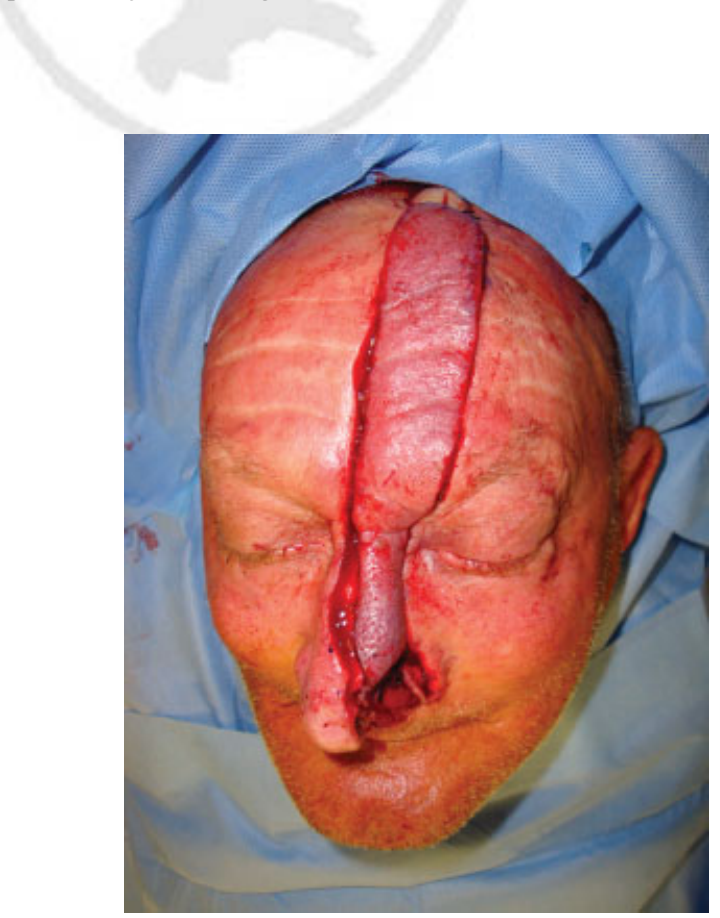

Fig. 19 The flap has been isolated. Some venous congestion is present. Again, this might happen frequently but will eventually resolve without consequences.

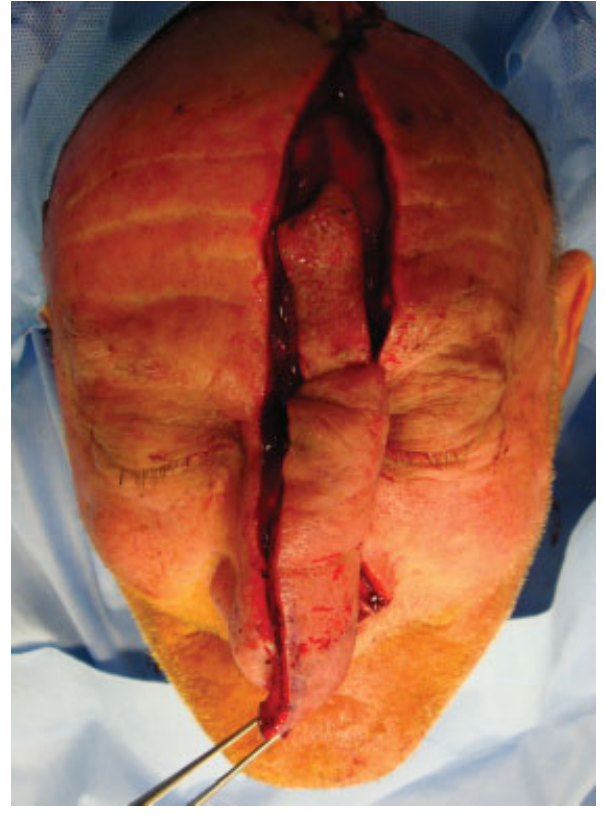

Fig. 20 After rotation, the flap is much longer than needed. The excess portion is not trimmed this time but folded double to replace the inner lining.

to the additional skin that is resected when subunit reconstruction is planned for a small defect. In case of distal defects, the dorsal nasal skin can also be used for inner lining reconstruction. Differently than other one-stage techniques, the contour is preserved because there is no additional soft tissue in the frontonasal angle and the pivot point is deeper than the glabella. The 180 degrees propeller rotation, as proved by our experience over the past years, if properly planned and executed, is safe, especially in the head and neck vascular territories, and even in patients with comorbidities or smokers like the population presented. In conclusion, STAAP flap is a good option for one-stage nose reconstruction

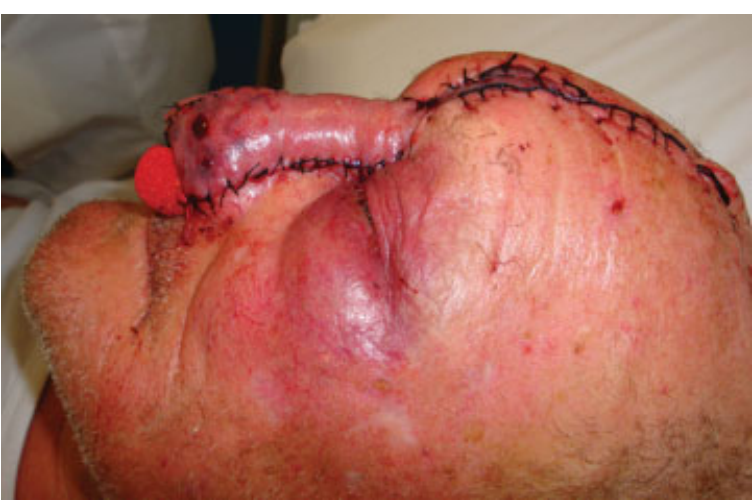

Fig. 21 Congestion might worsen at the end of the operation. Although it might seem quite scary, it will fade away. 


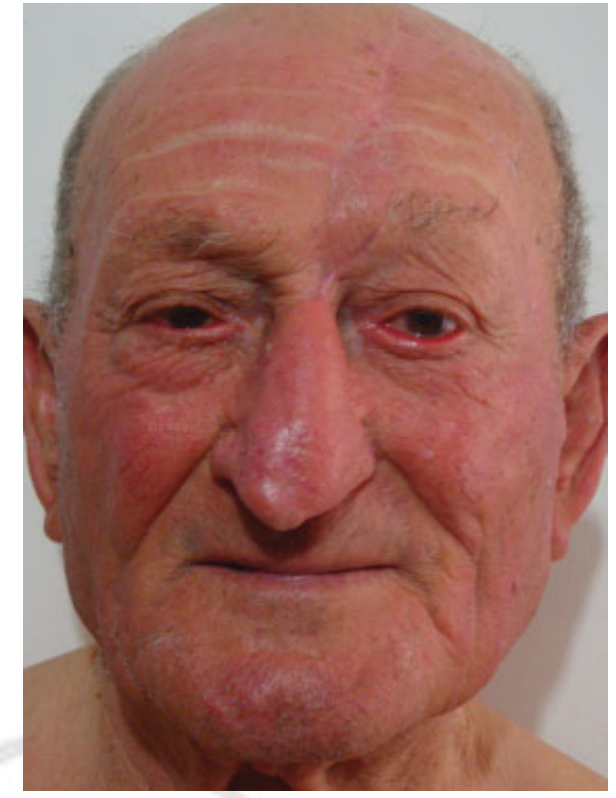

Fig. 22 One year postoperative frontal view. Despite congestion, the flap survived completely. Despite the flap was taken very narrow around the pedicle, the patient requested and underwent a correction in the glabellar region to get rid of the excess tissue. There result is a linear scar in the glabellar region that connects the nasal portion and the frontal portion of the flap.

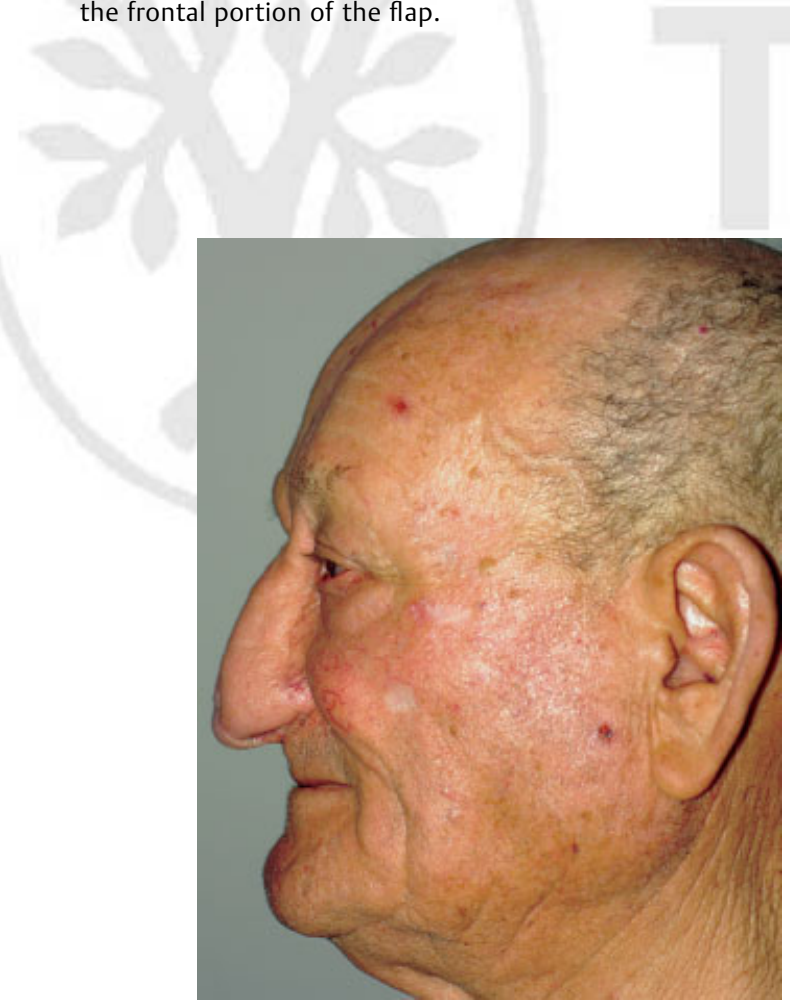

Fig. 23 One year postoperative lateral view.

and it is simpler than other single-stage techniques already published. To make a comparison between STAAP and threestage forehead flap has no sense because the STAAP is considered if an immediate healing is required. Because subunit reconstruction is necessarily less accurate, ${ }^{15}$ less-

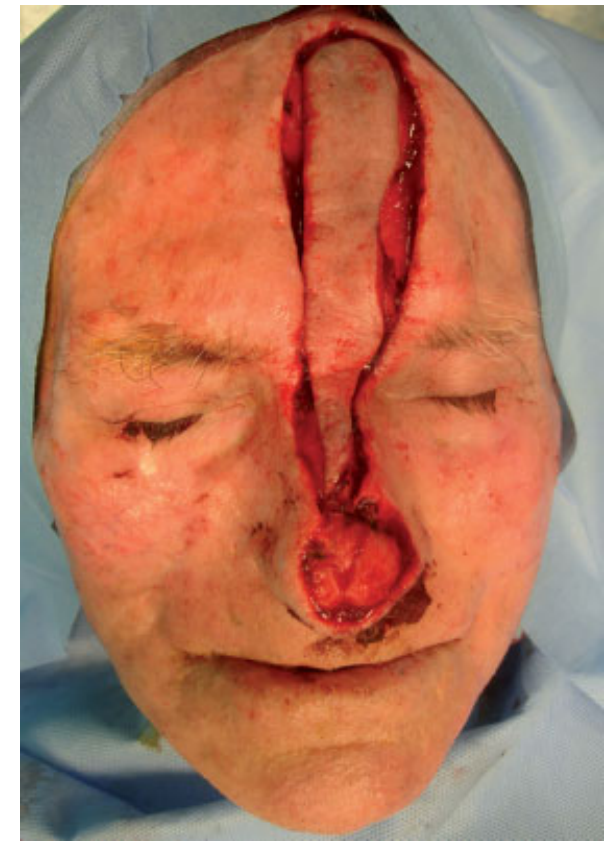

Fig. 24 Intraoperative view. After tumor excision the STAAP flap is harvested. The dorsal nasal skin interposed between the pedicle and the defect is harvested with the flap. STAAP, supratrochlear artery axial propeller.
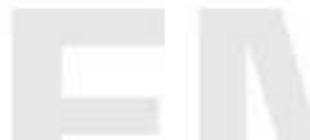

than-optimal cosmetic outcome can be achieved in only one stage. For this reason, in young and motivated people, the forehead flap is the first choice technique. However, results can be good as well (-Figs. 5-8) avoiding multiple operations and considerable efforts. This technique has become the first choice in our department for the selected group of patients whose priorities shift from cosmesis to fast recovery and less effort.
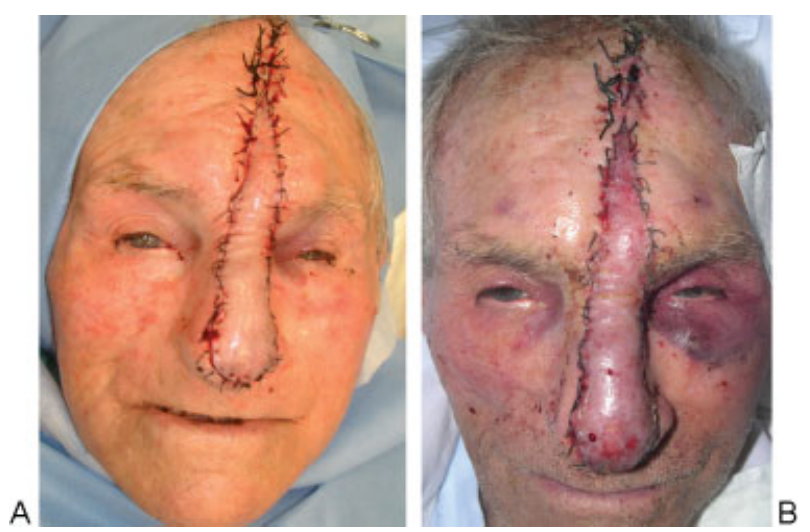

Fig. 25 (A) Immediate postoperative view. The color of the flap is good and capillary refill is normal. (B) Second postoperative view, picture taken at the bedside. Although the flap looked perfect at the end of the operation, sign of venous congestion developed. This should not be a cause of concern as these flaps are very forgiving and will eventually survive completely. 


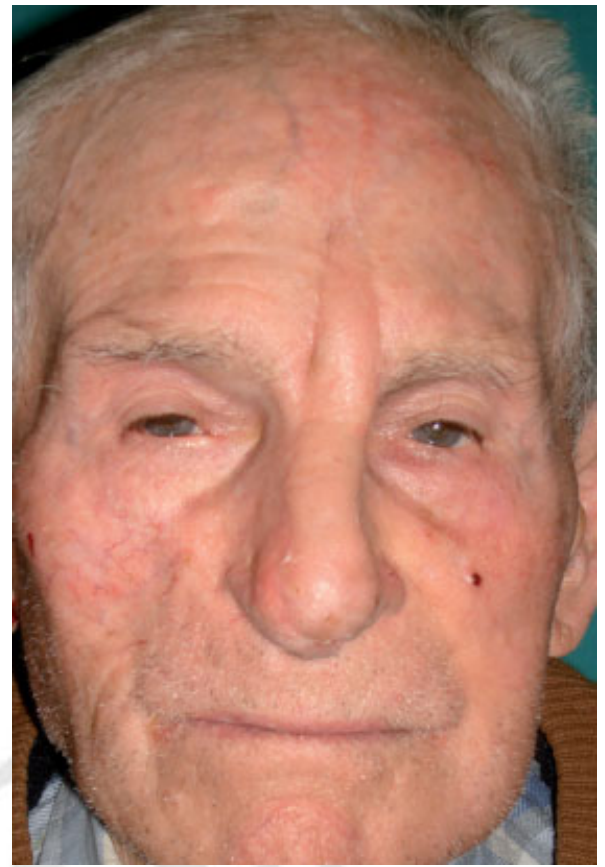

Fig. 26 Postoperative view after 1 year. On frontal view, the dorsal nasal skin is seen between the two eyebrows. Preserving this skin has a double advantage: it avoids pedicle compression-that can happen if it is discarded and the wound is closed primarily-and maintains the distance between the two eyebrows.

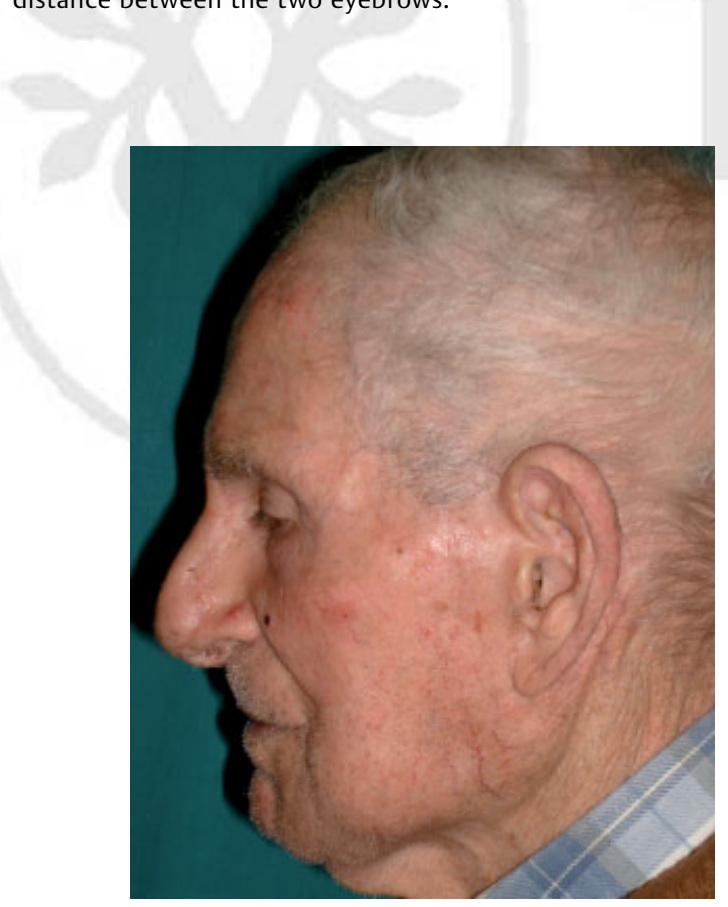

Fig. 27 Postoperative view after 1 year. On lateral view, preservation of the nasal contour is evident. The fullness at the nasion that is observed with other one-stage techniques such as the tunneled forehead flap is not seen with this technique because the skin at the root of the nose is not displaced but just turned 180 degrees.

\section{Conflict of Interest \\ None declared.}

\section{References}

1 Menick FJ. A 10-year experience in nasal reconstruction with the three-stage forehead flap. Plast Reconstr Surg 2002;109(6): 1839-1855, discussion 1856-1861

2 Menick FJ. Nasal reconstruction. Plast Reconstr Surg 2010;125(4): 138e-150e

3 Geddes CR, Morris SF, Neligan PC. Perforator flaps: evolution, classification, and applications. Ann Plast Surg 2003;50(1):90-99

4 Yang D, Tang M, Geddes CR, Morris SF. Vascular anatomy of the integument of the head and Neck. In: Blondeel PN, Morris SF, Hallock GG, Neligan PC, eds. Perforator Flaps Anatomy, Tecknique and Clinical Applications. St. Louis, MO: QMP; 2006:134-160

5 Hyakusoku H, Yamamoto T, Fumiiri M. The propeller flap method. Br J Plast Surg 1991;44:53-54

6 Pignatti M, Pasqualini M, Governa M, Bruti M, Rigotti G. Propeller flaps for leg reconstruction. J Plast Reconstr Aesthet Surg 2008; 61(7):777-783

7 D’Arpa S, Cordova A, Pirrello R, Moschella F. Free style facial artery perforator flap for one stage reconstruction of the nasal ala. J Plast Reconstr Aesthet Surg 2009;62(1):36-42

8 D'Arpa S, Cordova A, Pirrello R, Moschella F. One-stage reconstruction of the nasal ala: the free-style nasolabial perforator flap. Plast Reconstr Surg 2009;123(2):65e-66e

9 Cordova A, Di Lorenzo S, Moschella F. "Composite graft": a simple option for nasal lining. Int J Dermatol 2007;46(4):417-421

10 Converse JM, Wood-Smith D. Experiences with the forehead island flap with a subcutaneous pedicle. Plast Reconstr Surg 1963; 31:521-527

11 Park SS. The single-stage forehead flap in nasal reconstruction: an alternative with advantages. Arch Facial Plast Surg 2002;4(1): 32-36

12 Mombaerts I, Gillis A. The tunneled forehead flap in medial canthal and eyelid reconstruction. Dermatol Surg 2010;36(7):1118-1125

13 Cordova A, D'Arpa S, Moschella F. A new one-stage method for nose reconstruction: the supratrochlear artery perforator propeller flap. Plast Reconstr Surg 2012;129(3):571e-573e

14 Pignatti M, Ogawa R, Hallock GG, et al. The "Tokyo" consensus on propeller flaps. Plast Reconstr Surg 2011;127(2):716-722

15 Rohrich RJ, Griffin JR, Ansari M, Beran SJ, Potter JK. Nasal reconstruction-beyond aesthetic subunits: a 15-year review of 1334 cases. Plast Reconstr Surg 2004;114(6):1405-1416, discussion 1417-1419 